\title{
Characterisation of Feature Points in Eye Fundus Images
}

\author{
D. Calvo, M. Ortega, M.G. Penedo, and J. Rouco \\ VARPA Group, Department of Computer Science, University of A Coruña, Spain \\ $\{$ dcalvo,mortega, mgpenedo, jrouco\}@udc.es
}

\begin{abstract}
The retinal vessel tree adds decisive knowledge in the diagnosis of numerous opthalmologic pathologies such as hypertension or diabetes. One of the problems in the analysis of the retinal vessel tree is the lack of information in terms of vessels depth as the image acquisition usually leads to a $2 \mathrm{D}$ image. This situation provokes a scenario where two different vessels coinciding in a point could be interpreted as a vessel forking into a bifurcation. That is why, for traking and labelling the retinal vascular tree, bifurcations and crossovers of vessels are considered feature points. In this work a novel method for these retinal vessel tree feature points detection and classification is introduced. The method applies image techniques such as filters or thinning to obtain the adequate structure to detect the points and sets a classification of these points studying its environment. The methodology is tested using a standard database and the results show high classification capabilities.
\end{abstract}

Keywords: Feature points, classification of features, retinal images.

\section{Introduction}

In the field of medical diagnosis and disease study, it is necessary to analyse in detail medical images. This analysis usually covers the measuring of parameters, the calculation of values according to the image, and the monitoring of the structures. These tasks are usually performed manually by experts. This specialised process, takes up a lot of time and, as the task is done manually, is sensitive to subjective errors. It is, therefore, necessary to use more reliable methods.

The vascular tree of the retina can show morphological variations due to diseases or even aging. The branches intertwine, creating points where several vessels coincide. These points are of special importance in terms of analysis of the tree as, depending whether they are in the same spatial plane or not, they can be physically connected or otherwise just appear to be, due to the perspective of the image. These will be the feature points in this work.

Many methods for extracting information from the retina vessel tree can be found in the literature, but authors usually limit their work to a two dimensional extraction of the information. An analysis of the third dimension, depth, is needed. In the bibliography there are some works that try to solve this problem. For instance, the work proposed by Ali Can et al. [1 tries to solve the problem

E. Bayro-Corrochano and J.-O. Eklundh (Eds.): CIARP 2009, LNCS 5856, pp. 449 456. 2009.

(C) Springer-Verlag Berlin Heidelberg 2009 
in difficult images using the central vessel line to detect and classify the feature points. Other methods, like the proposed by Chia-Ling Tsai et al. 2], use vessel segments intersections as seeds to track vessel centre lines and classify feature points according to intersection angles. The work proposed by Enrico Grisan et al. 3] extracts the structure using a vessel tracking based method needing a previous step before detecting feature points to fix the loss of connectivity in the intersections. Another work, the proposed by V.Bevilacqua et al. 4, uses a small window to analyse the whole skeleton of the vascular structure. The main problem of this solution is the misclassification of crossovers, as they are only properly classified when the vessel segments intersect exactly in the same pixel.

This paper proposes a method to detect feature points of the retinal vascular tree and a subsequent classification of the detected points in two classes, bifurcations and crossovers. From an image of the retinal structure of the eye, the vascular tree is segmented. From this segmentation the skeleton is obtained, where the feature points are detected. In the last step, these feature points are classified according to a local analysis and a topological study.

The paper is organised as follows: in section 2 the segmentation process is presented. Section 3 describes the feature point detection method. In section 4 a description of the classification method used is presented. Section 5 shows the experimental results and validation obtained using standard retinal image databases. Finally, section 6 provides some discussion and conclusions.

\section{Arteriovenous Structure Segmentation}

Feature point detection implies an analysis of the vascular structure so a segmentation of the retinal vessel tree is required. In this work we use an approach with a particularly high sensitivity and specificity at classifying points as vessel or non vessel points. This segmentation process is done in two main steps: vascular structure enhancement and extraction of the arteriovenous tree.

By performing an initial enhancement the causes of a potential malfunction of the whole process, such as noise or vessel reflections are eliminated.

The preprocessing step applies a Tophat filter [5] to enhance the biggest and darkest structures present in the image, corresponding the vessels. Then, a median filter is applied to reduce noise and to tone down vascular reflex.

The vessel enhancement step uses a multiscalar approximation where the eigenvalues of the Hessian matrix [6] are used to apply a filter process that detects different sized geometric tubular structures. A function $B(p)$ is defined to measure the membership of a pixel, $p$, to vessel structures:

$$
B(p)=\left\{\begin{array}{cl}
0 & \text { if } \quad \lambda_{2}<0 \\
\exp \left(-2 R_{b}^{2}\right)\left(1-\exp \left(-\frac{S^{2}}{2 c^{2}}\right)\right) &
\end{array}\right.
$$

where $R_{b}=\frac{\lambda_{1}}{\lambda_{2}}$ (one and two eigenvalue), $c$ is the half of the max hessian norm, $\mathrm{S}$ represents a measure to "second order structures". Vessel pixels are characterised by a small $\lambda_{1}$ value and a higher positive $\lambda_{2}$ value. 
Once the blood vessels are enhanced, the vascular extraction is done in two steps: first an early segmentation and, second, a removal of isolated pixels.

An hysteresis based thresholding is done in the segmentation task. A hard threshold $\left(T_{h}\right)$ obtains the pixels with a high confidence of being vessel pixels while the weak threshold $\left(T_{w}\right)$ keeps all the pixels of the tree, even spurious ones. The final segmentation will be formed by all the pixels selected by the weak threshold connected to, at least, one pixel obtained by the hard threshold. $T_{h}$ and $T_{w}$ are obtained from two image properties: the percentage of image representing vessels and the percentage of the image classified as fundus. The gap between both percentages will include all not classified pixels. After calculating the percentiles with Equation 2 obtaining the values for the thresholds is immediate.

$$
P_{k}=L_{k}+\frac{k\left(\frac{n}{100}\right)-F_{k}}{f_{k}} * c, k=1,2, \ldots, 99
$$

where $L_{k}$ is percentile $\mathrm{k}$ lower limit, $n$ stands for the size of data set, $F_{k}$ is the accumulated frequency for $k-1, f_{k}$ represents the frequency of percentile $\mathrm{k}$ and $c$ is the size of the percentile interval ( 1 in this case).

To be able to obtain adequate results not only in high quality images from healthy eyes but also in poor images or images from eyes with diseases a last step is taken to erase spurious structures that, not belonging to the vascular structure, reached this point. To solve this, all isolated structures smaller than a prefixed number of pixels are erased. Figures 1(a) and 1(b) show, respectively, the original and segmented image.

Segmentation method was validated over 40 images from DRIVE [7] database using a $15 \times 15$ window for the Tophat filter and reaching a precision of $95 \%$.

\section{$3 \quad$ Feature Point Detection}

A feature point can be defined as every non internal vessel point. Specifically, feature points, are vessel crossovers, bifurcations or end points. The goal in this first stage is to detect the feature points of the retinal vessel tree. It is clear that, in the segmented image (Figure 1(b) , some properties are not constant along all the structure, like vessel width that decreases as the branch level of the structure becomes deeper. To unify this property, it is needed a method to reduce vessel width to one pixel without changing direction or connectivity. The skeleton is the structure that meets all these properties.

The results of the segmentation process force a previous preprocessing step before the skeletonization.Fig 1(b) shows gaps inside the segmented vessels that would give a wrong skeleton structure creating false feature points. To avoid these spurious feature points it is necessary to "fill" the gaps inside the vessels. For this, a dilation process using a modified median filter, to avoid erosion, is applied making the lateral vessel borders grow towards the centre filling the mentioned gaps. To "fill" as much white gaps as possible the dilation process is applied several times. The value of $N$ depends on the spatial resolution of the 


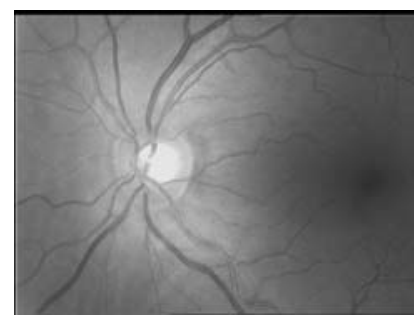

(a)

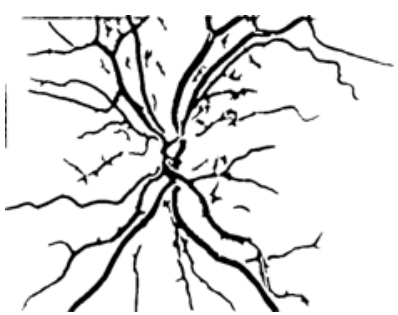

(c)

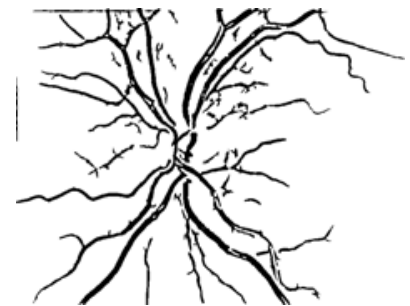

(b)

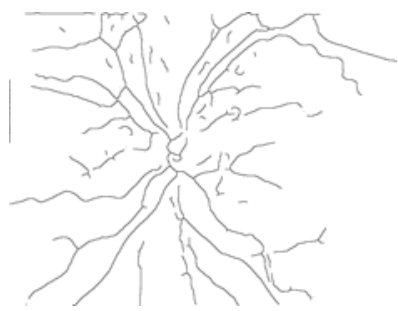

(d)

Fig. 1. (a) Original image (b) segmented image, (c) result of the dilation process with $N=4$ and (d) skeletonized vascular tree

images used, with the images used in this work $(768 \times 584)$ it was determined empirically that optimal values for $\mathrm{N}$ were around 4 (Fig $1(\mathrm{c})$.

Representing the skeleton by the medial axis function(MAF), defined as the set of points centre of the maximum radius circles that fit inside the vessels, is a very heavy task so template based method, versatiles and effectives, are applied to the segmented image. In this work the Stentiford thinning method [8] is used. Fig 1(d) shows the skeletonization results.

\subsection{Feature Points Location}

The feature point location is done according to the local information of the points. so an analysis of the neighbours of each point is done. According to the intersection number, $I(V)$, calculated for each point, $V$, as shown in 3 each point will be marked as an end point when $I(V)=1$, internal point when $I(V)=2$ and crossover or bifurcation when $I(V)>2$.

$$
I(V)=\frac{1}{2}\left(\sum_{i=1}^{8}\left|N_{i}(V)-N_{i+1}(V)\right|\right)
$$

where, $N_{i}(V)$ are the neighbours of the analysed point $V$ named clockwise consecutively.

Skeletonization process forces a pruning step to erase small artificial branches that create spurious feature points. Branches, understood as vessel segments between an end point and another feature point, are tracked and erased if smaller than the maximum vessel width expected in the image, $\zeta$. 
For the evaluation of the feature point detection process a set of 30 images from VARIA [9] database is used. The system obtained a value of sensitivity of $89.7 \%$ while only detecting a total of 32 false positives for a total of 662 true positives.

\section{Feature Point Classification}

The method used in 4 for the classification between bifurcations and crossovers, similar to the one used in this work for the detection, can lead to an incorrect classification of crossovers when, due to the angle and width of interwining vessels, not all vessel segments coincide in the same skeleton pixel. To solve this problem and produce a robust and valid classification a further analysis, according to local and topological features, for points with $I(V)>2$ is done.

The first classification step is done according to local features of the points without considering, for it, the effect of the other points. Each detected feature point, $F$, is used as centre of a circumference with radius $R_{c}$ used for the analysis. $n(F)$ gives the number of vessel segments that intersect the circumference where $n(F)=3$ corresponds to a bifurcation and $n(F)=4$ to a crossover. Fig 2 shows the blood vessels, the circumference used to do the analysis, and, coloured darker, the pixels where the vessels intersect the circumference.

To avoid missclasifications when the circumference is intersected by vessels alien to the analysed point, a vote system with three radius is used. Two values, $\mathrm{C}(\mathrm{F})$ and $\mathrm{B}(\mathrm{F})$, meaning the number of votes for a point $\mathrm{F}$ to be classified, respectively, as a crossover and a bifurcation are used:

$$
\begin{aligned}
& C(F)=2 * C\left(F, R_{1}\right)+C\left(F, R_{c}\right)+C\left(F, R_{2}\right) \\
& B(F)=B\left(F, R_{1}\right)+B\left(F, R_{c}\right)+2 * B\left(F, R_{2}\right)
\end{aligned}
$$

where $C\left(F, R_{i}\right)$ and $B\left(F, R_{i}\right)$ are binary values indicating if $F$ is classified, respectively, as a crossover or a bifurcation using a radius $R_{i}, R_{1}=R_{c}-\rho$ and $R_{2}=R_{c}+\rho$, with $\rho$ a fixed amount, are the radius around $R_{c}$. Note that the contribution of the small radius is more valuable, and therefore weighted, in the crossover classification while for bifurcations the big radius adds more information. $F$ will be classified as a crossover when $C(F)>B(F)$ and a bifurcation otherwise.

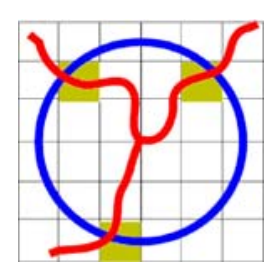

(a)

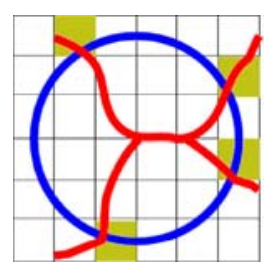

(b)

Fig. 2. Preliminary feature point classification according to the number of vessel intersections where (a) represents a bifurcation and (b) a crossover 
Due to the representation of crossovers in the skeleton, this information is not enough to assure that a feature point is a crossover while being a necessary condition. According to this, a topological classification is needed analysing the feature points in pairs, $\left(F_{1}, F_{2}\right)$, attending to their Euclidean distance $d\left(F_{1}, F_{2}\right)$. If both $F_{1}$ and $F_{2}$ are connected by a vessel segment and $d\left(F_{1}, F_{2}\right)<=2 * R_{c}$, both points are merged into a crossover in the middle point between them.

Not classified real crossovers would create two false bifurcations in the final result. Thus, another threshold, $R_{b}$, is needed to decide which points are accepted as bifurcations. For each pair of bifurcations, understood as two connected bifurcations that minimise their Euclidean distance, a circumference with radius $R_{b}$ centred in the middle point between them is used. This circumference cannot contain both points. So, points not fulfilling the conditions are marked as not classified. Note that $R_{c}$ and $R_{b}$ parameters allow to tune the system in terms of specificity and sensitivity as some domains would require different performances. In the next section, some experiments and performance results are shown.

\section{$5 \quad$ Results}

For the analysis of the methodology a set of 45 images randomly extracted from VARIA [9] and labelled by experts were used. These images are centred in the optic disc with spatial resolution of $768 \times 584$ pixels.

Image preprocessing parameters are necessary to the correct performance of later steps. For the image set used, the adequate number of dilations, $N$, is four, the chosen prune threshold is $\zeta=20$ and the radius around $R_{c}$ in the vote system, $\rho=5$.

$R_{c}$ and $R_{b}$ allow to tune the specificity and sensitivity of crossover and bifurcation classification respectively, so a quantitative study according to these parameters is presented. The results allow to choose the adequate parameters for a specific domain where the desired sensitivity or specificity levels can change depending on the False Positives, True Positives and False Negatives as shown in Fig $3(\mathrm{a})$,

The figure shows how the number of correct classified crossovers increase with the radius size. This tendency could throw the idea of increasing the radius size until obtaining a big number of classified crossovers, however, increasing the radius also increases the number of misclassified crossovers.

Fig 3(b) shows the results for bifurcation classification according to the chosen $R_{b}$ radius. This figure displays a new category, the non classified points, that includes the points that fulfilling the morphology conditions are not close enough to be classified as crossover and not far enough not to be classified as independent bifurcations. The bigger radius, $R_{b}$, used the more number of points without classifying but the number of false positives will be below the $1 \%$. Opposite to this, if a big level of true positives is needed with a small radius the sensitivity is over the $70 \%$. Selecting $R_{c}=10$ and $R_{b}=30$, the global sensitivity of the system is $75 \%$ and the specificity $93 \%$. 


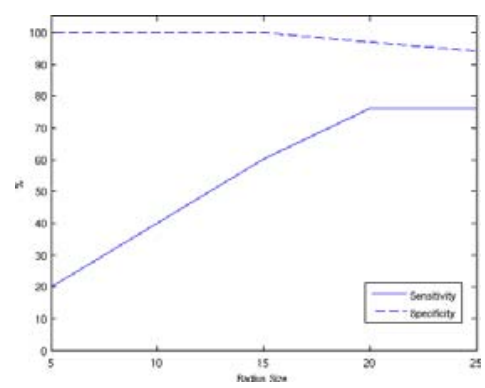

(a)

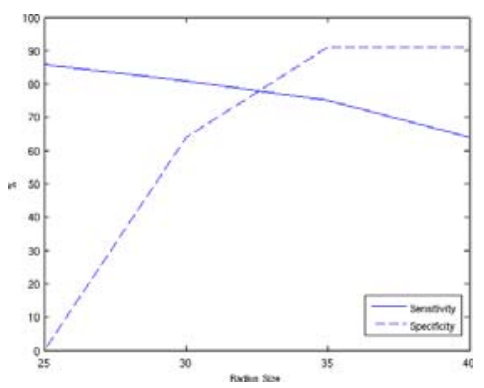

(b)

Fig. 3. Analysis of the influence of $R_{c}$ for the crossovers, (a), and $R_{b}$ for the bifurcations, (b) in the classification performance of the system

Table 1. Obtained results compared to the results given in $[3$

\begin{tabular}{|l|c|c|c|c|}
\cline { 2 - 5 } \multicolumn{1}{c|}{} & \multicolumn{2}{c|}{ Bifurcations } & \multicolumn{2}{c|}{ Crossovers } \\
\cline { 2 - 5 } \multicolumn{1}{c|}{} & Sensitivity & Specificity & Sensitivity & Specificity \\
\hline D. Calvo et al. & $75 \%$ & $91 \%$ & $76 \%$ & $96 \%$ \\
\hline E. Grisan et al. & $76 \%$ & $87 \%$ & $62 \%$ & $74 \%$ \\
\hline
\end{tabular}

As said in Section 3, the technique shown in [4] has the problem of the crossover misclassification due to the skeleton representation where a crossover turns into two close bifurcations. This paper, 4, does not offer quantitative results but our implementation of this technique shows that nearly every point is classified as a bifurcation, being capable to classify correctly only $3 \%$ of the crossovers. The work proposed in 3 extracts the structure using a vessel tracking based with the results shown in Table 1. Other previous techniques do not offer quantitative results in the characterisation task to compare with. The main improvement comes in the crossover rate, due to the radius proposed. In general, the system exhibits a very high specificity rate for both classes making it suitable for critical tasks.

\section{Conclusions and Future Work}

In this work a method for the detection and classification of the feature points of the retinal vascular tree using several image processing techniques has been presented. The detection and classification of these points is important because it increases the information about the retinal vascular structure. Having the feature points of the tree allows an objective analysis of the diseases that cause modifications in the vascular morphology.

To improve the system a future work could be use vessel features to classify the feature points. The classification method is done now according to the number of vessels that belong to the point and in the relationship between pairs of 
points. The presented work is able to be applied in many other domains such as authentication task, using retinal images in order to help the comparison between points according to the classification given by this method.

\section{Acknowledgements}

This paper has been partly funded by the Ministerio de Ciencia y Tecnología. Instituto de Salud Carlos III through the grant contract PI08/90420.

\section{References}

1. Can, A., Shen, H., Turner, J., Tanenbaum, H., Roysam, B.: Rapid automated tracing and feature extraction from retinal fundus images using direct exploratory algorithms. IEEE Transactions on Information Technology in Biomedicine 3(2), 125-138 (1999)

2. Tsai, C.L., Stewart, C., Tanenbaum, H., Roysam, B.: Model-based method for improving the accuracy and repeatability of estimating vascular bifurcations and crossovers from retinal fundus images. IEEE Transactions on Information Technology in Biomedicine 8(2), 122-130 (2004)

3. Grisan, E., Pesce, A., Giani, A., Foracchia, M., Ruggeri, A.: A new tracking system for the robust extraction of retinal vessel structure. In: IEMBS 2004. 26th Annual International Conference of the IEEE, September 2004. Engineering in Medicine and Biology Society, vol. 1, 3, pp. 1620-1623 (2004)

4. Bevilacqua, V., Cambó, S., Cariello, L., Mastronardi, G.: A combined method to detect retinal fundus features. In: Proceedings of IEEE European Conference on Emergent Aspects in Clinical Data Analysis (2005)

5. Dougherty, E.R.: Mathematical morphology in image processing / edited by Edward Dougherty. M. Dekker, New York (1993)

6. Frangi, A.F., Niessen, W.J., Vincken, K.L., Viergever, M.A.: Multiscale vessel enhancement filtering, $130+(1998)$

7. Drive: Digital retinal images for vessel extraction, http://www.isi.uu.nl/Research/Databases/DRIVE/

8. Stentiford, F.W.M., Mortimer, R.G.: Some new heuristics for thinning binary handprinted characters for ocr. IEEE Transactions on Systems, Man and Cybernetics 13(1), 81-84 (1983)

9. Varia: Varpa retinal images for authentication, http://www.varpa.es/varia.html 